\title{
A hybrid type I trial to increase Veterans' access to insomnia care: study protocol for a randomized controlled trial
}

\author{
Adam D. Bramoweth ${ }^{1,2^{*}}$, Anne Germain ${ }^{3}$, Ada O. Youk ${ }^{1,4}$, Keri L. Rodriguez ${ }^{1,5}$ and Matthew J. Chinman 1,2,6
}

\begin{abstract}
Background: Chronic insomnia is among the most reported complaints of Veterans and military personnel referred for mental health services. It is highly comorbid with medical and psychiatric disorders, and is associated with significantly increased healthcare utilization and costs. Evidence-based psychotherapy, namely Cognitive Behavioral Therapy for Insomnia (CBTI), is an effective treatment and recommended over prescription sleep medications. While CBTI is part of a nationwide rollout in the Veterans Health Administration to train hundreds of providers, access to treatment is still limited for many Veterans due to limited treatment availability, low patient and provider knowledge about treatment options, and Veteran barriers such as distance and travel, work schedules, and childcare. Uptake of a briefer, more primary-care-friendly treatment into routine clinical care in Veterans Affairs (VA) primary care settings, where insomnia is typically first recognized and diagnosed, may effectively and efficiently increase access to effective insomnia interventions and help decrease the risks and burdens related to chronic insomnia.

Methods: This hybrid type I trial is composed of two aims. The first preliminarily tests the clinical non-inferiority of Brief Behavioral Treatment for Insomnia (BBTI) versus the current "gold standard" treatment, CBTI. The second is a qualitative needs assessment, guided by the Consolidated Framework for Implementation Research (CFIR), to identify potential factors that may affect successful implementation and integration of behavioral treatments for insomnia in the primary care setting. To identify potential implementation factors, individual interviews are conducted with the Veterans who participate in the clinical trial, as well as VA primary care providers and nursing staff.

Discussion: It is increasingly important to better understand barriers to, and facilitators of, implementing insomnia interventions in order to ensure that Veterans have the best access to care. Furthermore, it is important to evaluate the potential for new avenues of treatment delivery, like BBTI in the primary care setting, which can benefit Veterans who may not have adequate access to specialty mental health providers trained in CBTI.
\end{abstract}

Trial registration: ClinicalTrials.gov, ID: NCT02724800. Registered on 31 March 2016.

Keywords: Insomnia, Randomized controlled trial, Cognitive behavior therapy, Behavior therapy, Qualitative research, Veterans

\footnotetext{
*Correspondence: Adam.Bramoweth@va.gov

${ }^{1}$ Center for Health Equity Research and Promotion, VA Pittsburgh Healthcare

System, Research Office Building (151R-U), University Drive C, Pittsburgh, PA

15240, USA

${ }^{2}$ Mental Illness Research, Education and Clinical Center, VA Pittsburgh

Healthcare System, Research Office Building (151R-U), University Drive C,

Pittsburgh, PA 15240, USA

Full list of author information is available at the end of the article
}

(c) The Author(s). 2018 Open Access This article is distributed under the terms of the Creative Commons Attribution 4.0 International License (http://creativecommons.org/licenses/by/4.0/, which permits unrestricted use, distribution, and reproduction in any medium, provided you give appropriate credit to the original author(s) and the source, provide a link to the Creative Commons license, and indicate if changes were made. The Creative Commons Public Domain Dedication waiver (http://creativecommons.org/publicdomain/zero/1.0/) applies to the data made available in this article, unless otherwise stated. 


\section{Background}

Chronic insomnia-difficulty initiating and maintaining sleep that persists for more than 3 months-is a prevalent disorder among adults, approximately $25 \%$, but can be particularly pervasive among military personnel and Veterans, with estimates nearing $75 \%$ in some samples [1-5]. Chronic insomnia is among the most reported complaints of Veterans [6] and is the most common initial complaint of military personnel referred for mental health services [1, 7]. Potential risk factors include deployment overseas, engaging in combat, 24 hours/7 days a week work schedules, adjusting to separation from military and reintegration to civilian life, as well as the numerous medical and mental health problems that commonly affect military personnel and Veterans [8-13]. It is also a risk factor for the development of depression [14] and metabolic and cardiovascular diseases [15]. Furthermore, insomnia is associated with significant healthcare utilization, and both individual and societal economic burden [16-19].

Despite the significant impact of insomnia, it remains undertreated [20]. When identified, it is most often treated in primary care with pharmacotherapy, rather than the first-line recommendation, Cognitive Behavioral Therapy for Insomnia (CBTI) [21, 22]. Pharmacotherapy is associated with risks of dependence, tolerance, and poorer quality sleep [23-25], whereas evidence-based psychotherapies for primary and comorbid insomnia results in better longterm outcomes, no drug dependence or polypharmacy risk, and potential cost savings [26, 27]. Based on our experience, numerous system-, provider-, and patient-level factors contribute to the gap between the high prevalence of insomnia and the relatively low use of CBTI, and potentially contribute to the high use of prescription medications: (1) shortage of CBTI-trained clinicians; (2) treatment restricted to mental health clinics; (3) insomnia being considered a symptom of another disorder; (4) lack of patient and provider knowledge regarding CBTI availability; (5) barriers to attend appointments such as distance to travel, work schedule, and childcare; and (6) burdensome duration and delivery method (CBTI can be six or more inperson, 45-min sessions).

The Department of Veterans Affairs (VA) nationwide CBTI rollout, which began in 2011, substantially increased the number of providers who can deliver evidence-based treatment with fidelity and helped to increase access to care. An evaluation of 696 Veterans who participated as part of the CBTI rollout found that $60 \%$ who completed treatment had insomnia severity reductions, per the Insomnia Severity Index (ISI), of $\geq 8$ points (mean change 20.7 to 10.9), with a pre- to post-treatment Cohen's $d$ effect size of $2.3[28,29]$. While the rollout has been successful to date and continues to train providers, CBTI is still only being delivered to a fraction of those who could benefit. Thus, in order to increase the viability of cognitive and/ or behavioral insomnia treatments in the VA, it is critical to not only determine which treatments are most effective, but also to determine which implementation factors (e.g., barriers and facilitators) most impact the uptake of these treatments by patients and providers in routine clinical practice. An evidence-based behavioral insomnia treatment that combines brevity (four sessions or fewer), multiple delivery modalities (in-person and phone), and is delivered by non-physician, non-sleep-specialist clinicians may help to overcome barriers associated with the current standard of care treatment, CBTI. However, the four weekly sessions of Brief Behavioral Treatment for Insomnia (BBTI) [30, 31] (two in-person, two phone calls), focused on the behavioral aspects of CBTI, have also proven to be efficacious among Veterans [32], and are potentially easier to implement in primary care settings because this approach is shorter and requires less training to deliver competently. Thus, BBTI could be an ideal intervention for delivery in the context of co-located, collaborative, integrated primary care within the VA, which employs a variety of providers of differing training levels.

Uptake of BBTI into primary care could effectively and efficiently increase access to insomnia treatment and potentially decrease some of the risks and burdens associated with chronic insomnia. However, it is necessary to determine whether BBTI offers non-inferior treatment outcomes to CBTI. Additionally, given that the implementation factors associated with BBTI and CBTI are not well known, it is also important to determine whether BBTI experiences fewer patient-, provider-, and system-level barriers to implementation than CBTI. Therefore, the current proposal utilizes a hybrid type I research design that includes: (1) a pilot comparative effectiveness trial of BBTI versus CBTI and (2) a qualitative needs assessment of healthcare provider- and Veteran-level implementation factors guided by the Consolidated Framework for Implementation Research (CFIR), the predominant model of implementation factors [33].

\section{Methods \\ Overview and study design}

This project is a hybrid type I, comparative effectiveness trial that compares BBTI to CBTI. The project is being conducted at one large, urban VA Medical Center (VAMC) over a 3-year period and is composed of two aims. The first aim is a randomized, non-inferiority trial to compare the effectiveness of BBTI versus CBTI. We hypothesize that (1) both BBTI and CBTI will significantly reduce insomnia symptoms, per the ISI, from pre- to posttreatment and (2) BBTI will be non-inferior to CBTI based on ISI change scores from pre- to post-treatment. Veterans with chronic insomnia are randomly assigned to either BBTI or CBTI with treatment being delivered by licensed psychologists and assessments at baseline, post-treatment, 3-month follow-up, and 12-month follow-up. See Fig. 1 for 


\begin{tabular}{|c|c|c|c|c|c|c|c|c|c|}
\hline \multirow[b]{3}{*}{ TIMEPOINT } & \multicolumn{9}{|c|}{ STUDY PERIOD } \\
\hline & \multirow{2}{*}{$\begin{array}{c}\text { Enrollment } \\
\begin{array}{c}\text {-2 weeks } \\
\left(t_{1}\right)\end{array} \\
\end{array}$} & \multicolumn{5}{|c|}{$\begin{array}{c}\text { Intervention } \\
\text { (weeks) }\end{array}$} & \multicolumn{3}{|c|}{$\begin{array}{c}\text { Follow-Up } \\
\text { Assessments }\end{array}$} \\
\hline & & 1 & 2 & 3 & 4 & 5 & $\begin{array}{c}+1 \\
\text { week } \\
\left(t_{2}\right) \\
\end{array}$ & \begin{tabular}{|c}
+3 \\
months \\
$(\mathrm{t} 3)$
\end{tabular} & \begin{tabular}{|c}
+12 \\
months \\
$\left(t_{4}\right)$
\end{tabular} \\
\hline \multicolumn{10}{|l|}{ Enrollment: } \\
\hline Informed consent & $\mathrm{X}$ & & & & & & & & \\
\hline DSM-5-SW & $\mathrm{X}$ & & & & & & & & \\
\hline SCID-5-RV & $\mathrm{X}$ & & & & & & & & \\
\hline SLUMS & $\mathrm{X}$ & & & & & & & & \\
\hline STOP-BANG & $\mathrm{X}$ & & & & & & & & \\
\hline Allocation & $\mathrm{X}$ & & & & & & & & \\
\hline \multicolumn{10}{|l|}{ Interventions: } \\
\hline CBTI & & $\mathrm{X}$ & $\mathrm{X}$ & $\mathrm{X}$ & $\mathrm{X}$ & $\mathrm{X}$ & & & \\
\hline BBTI & & $\mathrm{X}$ & $\mathrm{P}$ & $\mathrm{X}$ & $\mathrm{P}$ & & & & \\
\hline \multicolumn{10}{|l|}{ Assessments: } \\
\hline ISI & $\mathrm{X}$ & $\mathrm{X}$ & $\mathrm{X}$ & $\mathrm{X}$ & $X$ & $\mathrm{X}$ & $\mathrm{X} / \mathrm{M}$ & $\mathrm{X} / \mathrm{M}$ & $\mathrm{X} / \mathrm{M}$ \\
\hline Sleep Diaries & $\mathrm{X}$ & $\mathrm{X}$ & $\mathrm{X}$ & $\mathrm{X}$ & $X$ & $\mathrm{X}$ & $\mathrm{X} / \mathrm{M}$ & $\mathrm{X} / \mathrm{M}$ & $\mathrm{X} / \mathrm{M}$ \\
\hline PSQI & $\mathrm{X}$ & & & & & & $\mathrm{X} / \mathrm{M}$ & $\mathrm{X} / \mathrm{M}$ & $\mathrm{X} / \mathrm{M}$ \\
\hline DBAS & $\mathrm{X}$ & & & & & & $\mathrm{X} / \mathrm{M}$ & $\mathrm{X} / \mathrm{M}$ & $\mathrm{X} / \mathrm{M}$ \\
\hline ESS & $\mathrm{X}$ & & & & & & $\mathrm{X} / \mathrm{M}$ & $\mathrm{X} / \mathrm{M}$ & $\mathrm{X} / \mathrm{M}$ \\
\hline PHQ-9 & $\mathrm{X}$ & & & & & & $\mathrm{X} / \mathrm{M}$ & $\mathrm{X} / \mathrm{M}$ & $\mathrm{X} / \mathrm{M}$ \\
\hline GAD-7 & $\mathrm{X}$ & & & & & & $\mathrm{X} / \mathrm{M}$ & $\mathrm{X} / \mathrm{M}$ & $\mathrm{X} / \mathrm{M}$ \\
\hline PCL-5 & $\mathrm{X}$ & & & & & & $\mathrm{X} / \mathrm{M}$ & $\mathrm{X} / \mathrm{M}$ & $\mathrm{X} / \mathrm{M}$ \\
\hline Fatigue & $\mathrm{X}$ & & & & & & $\mathrm{X} / \mathrm{M}$ & $\mathrm{X} / \mathrm{M}$ & $\mathrm{X} / \mathrm{M}$ \\
\hline Global Health & $\mathrm{X}$ & & & & & & $\mathrm{X} / \mathrm{M}$ & $\mathrm{X} / \mathrm{M}$ & $\mathrm{X} / \mathrm{M}$ \\
\hline WSAS & $\mathrm{X}$ & & & & & & $\mathrm{X} / \mathrm{M}$ & $\mathrm{X} / \mathrm{M}$ & $\mathrm{X} / \mathrm{M}$ \\
\hline
\end{tabular}

Fig. 1 Schedule of enrollment, interventions, and assessments

the Standard Protocol Items: Recommendations for Interventional Trials (SPIRIT) guidelines; the full SPIRIT Checklist is available as Additional file 1. CBTI is a wellestablished, evidence-based psychotherapy with broad dissemination throughout the VA and is considered the recommended first-line treatment for insomnia per the National Institutes of Health and the American College of Physicians [22, 34]. Treatment outcomes for CBTI typically include significant reduction of symptoms per the ISI and Cohen's $d$ effect sizes $>1$ at post treatment [29]. The comparison treatment, BBTI, is also effective, with significant treatment outcomes similar to CBTI in active-duty military and Veterans' samples [30, 32]; however, the two have not yet been compared directly.

The second aim is a qualitative needs assessment, guided by the CFIR, to identify potential factors that may impact successful implementation and integration of behavioral and cognitive behavioral treatments for insomnia in the primary care setting. Given the under-diagnosis of insomnia and subsequent inadequate resources to treat insomnia with behavioral and cognitive behavioral treatments, the qualitative needs assessment may identify key perceived barriers and facilitators to implementing these treatments in primary care, a setting that may be able to significantly increase access to treatment, especially when it is not available in other settings such as mental health and/or sleep medicine. Individual interviews are conducted with the Veterans who participate in the clinical trial, as well as primary care providers and nursing staff.

\section{Procedures, participants, measures, and analyses by study aim}

Aim 1: non-inferiority, comparative effectiveness trial of CBTI versus $\mathrm{BBTI}$

\section{Data source}

Fifty-six Veterans with chronic insomnia will be randomized to either of the two treatment arms, BBTI (active comparison) or CBTI (reference treatment).

\section{Recruitment and participants}

The target population is a clinical sample of Veterans with chronic insomnia (see Table 1). Veterans are eligible if they meet the diagnostic criteria for an insomnia disorder according to the Diagnostic and Statistical Manual of Mental Disorders-5 (DSM-5) and are 18 years of age or older. In an attempt to be as pragmatic and reflective of typical clinical samples, exclusionary criteria were limited to key factors that would likely prevent a Veteran from engaging in similar treatments for insomnia in a typical VA setting. Veterans are excluded if they have a disorder that would 
Table 1 Inclusion and exclusion criteria

Inclusion criteria
Military Veteran
Age $\geq 18$ years
$|\mathrm{S}| \geq 15$ (moderately severe insomnia) and DSM-5 criteria for insomni
disorder
Exclusion criteria

Current/past bipolar disorder or seizure disorder, and current psychotic disorder in order to avoid potential exacerbation of manic/ hypomanic, seizure, and psychotic symptoms, respectively, as adverse reactions to aspects of $\mathrm{BBTI} / \mathrm{CBTI}$ (e.g., sleep restriction)

Current alcohol use disorder or substance use disorder as BBTI/CBTI cannot reverse the adverse effects of substances on sleep

Other current, severe or unstable, psychiatric and medical disorders that necessitate clinical management that can confound results (e.g. cancer [receiving chemotherapy], suicidality, recent hospitalization [medical/surgical] for which recovery overlaps with study onset and duration, open skull/brain injury, moderate to severe traumatic brain injury)

Previously diagnosed sleep apnea that is not adequately treated or probable sleep apnea (STOP-BANG $\geq 5$ )

Moderate to severe cognitive impairment (SLUMS $\leq 20)$ and/or diagnosis in the medical record indicative of moderate-to-severe cognitive impairment

If using a sleep medication, the medication and dose has changed in the past month and/or is expected to change during the treatment phase of the study

If using other psychotropic medication, medication and dose has changed in the past 2 months and/or are expected to change during the treatment phase of the study

The following are exclusionary as they represent (potentially) temporary states/situations that may significantly impair normal sleep:

Women who are pregnant and/or breast feeding

Unstable environment that is not in one's control (e.g., homeless, temporary group home, care-taking duties at night)

Shift work: severe delayed sleep phase disorder (e.g., habitual bedtime after 4 a.m. or habitual rise time after 11 a.m.)

Restless leg syndrome with symptoms $>2$ times per week and causing significant distress

BBTI Brief Behavioral Treatment for Insomnia, CBTI Cognitive Behavioral Therapy for Insomnia, DSM Diagnostic and Statistical Manual of Mental Disorders, ISI Insomnia Severity Index, SLUMS St. Louis University Mental Status Examination

significantly increase their risk of experiencing side effects with standard treatment or would require a significant adaptation of treatment. Veterans are also excluded consistent with the standard of care for cognitive and/or behavioral treatment of insomnia at the study VAMC.

\section{Screening process}

The screening interview, conducted by the study coordinator, includes gathering Veteran information regarding: demographics (age, sex, and Veteran status); clinical information, including a brief description of sleep complaint and the ISI $(\geq 15$, indicative of at least moderately severe insomnia); exclusionary DSM-5 diagnoses (e.g., psychotic disorders); known medical and sleep disorders; and current alcohol and drug use. This information is used to determine preliminary eligibility and those Veterans who are eligible are invited to a face-to-face visit to complete informed consent, a brief cognitive screen, a diagnostic assessment for sleep and psychiatric disorders, and collection of baseline measures. Ineligible Veterans, if interested, are referred to an appropriate clinic (e.g., behavioral health, sleep medicine, insomnia clinic). Once a participant is screened, they are assigned a unique study identification number that is used on all study documents in order to maintain confidentiality.

\section{Baseline screening measures and assessments}

The initial face-to-face visit includes answering Veterans' questions and addressing concerns, and administering written informed consent and baseline assessments and measures. The study coordinator administers written informed consent and conducts the baseline assessment unless otherwise indicated.

In order to assess for insomnia disorder, inclusionary criteria, as well as other sleep disorders that may be exclusionary, the STRONG STAR Clinical Interview for DSM-5 Sleep-Wake Disorders (DSM-5-SW [35]) is used. The STRONG STAR Interview, developed by the South Texas Research Organizational Network Guiding Studies on Trauma and Resilience (STRONG STAR), was developed to assess for all DSM-5 Sleep-Wake disorders, including: insomnia, hypersomnia, circadian rhythm disorders, obstructive sleep apnea, restless leg syndrome, periodic limb movements, parasomnias, nightmares, rapid eye movement (REM) sleep behaviors, and narcolepsy. To further screen for the presence of untreated obstructive sleep apnea, participants are administered the STOP-BANG Questionnaire [36], which is an eight-item self-report screening tool for obstructive sleep apnea, scored yes/no. Scores range $0-8$ with scores $\geq 5$ indicative of high risk of moderate-to-severe obstructive sleep apnea. Questions ask about snoring, daytime sleepiness, blood pressure, Body Mass Index, age, neck circumference, and gender. Veterans scoring $\geq 5$ are excluded from the study until further assessed by the study VAMC Sleep Medicine Clinic for sleep apnea or other breathing-related sleep disorders. Veterans with suspected non-insomnia sleep disorders are also referred to the Sleep Medicine clinic for further assessment. In the event that the Sleep Medicine Clinic evaluation rules out non-insomnia sleep disorders, the participant is eligible for the study.

To screen for potential exclusionary cognitive impairment, the St. Louis University Mental Status Examination (SLUMS [37]) is administered. The SLUMS is a brief cognitive impairment screening tool, validated in Veterans. Domains include: orientation; short-term memory recall; executive function; attention, concentration, and working 
memory; semantic fluency; and visuospatial ability. A score $\leq 20$, indicative of serious cognitive impairment or dementia, results in exclusion from the study.

In order to establish the presence of psychiatric disorders as baseline participant characteristics and also as potential variables to include in analyses, participants are administered the Structured Clinical Interview for DSM-5 Research Version (SCID-5-RV [38]). The SCID$5-\mathrm{RV}$ is a semi-structured interview guide for diagnosing DSM-5 disorders. It includes the major diagnostic categories and the diagnostic elements needed for inclusion and exclusion criteria. Assessing for a broad range of psychiatric disorders helps to better characterize the sample. The study principal investigator (PI), a licensed psychologist, conducts the SCID-5-RV assessment.

\section{Outcome measures}

The primary sleep outcome is the Insomnia Severity Index (ISI [39]). The ISI is a seven-item self-report measure of subjective insomnia severity, satisfaction with sleep, and daytime impairment. Items are rated 0-4; a cutoff of $\geq 15$ points reflects clinically significant insomnia and is the minimum score to be eligible for participation. At post treatment, a reduction of $\geq 8$ points indicates a moderate response to treatment; a score $\leq 7$ points indicates no clinical insomnia and combined with a reduction of $\geq 8$ points indicates treatment remission. The ISI is also administered at all in-person treatment sessions for both treatment arms. Since sleep is a multidimensional behavior, several additional self-report measures are used as secondary outcomes to assess changes pre to post treatment. We use Sleep Diaries [40] to measure sleep behaviors such as sleep onset latency (SOL), wake after sleep onset (WASO), sleep efficiency (SE), nighttime awakenings (NWAK), time in bed (TIB), total sleep time (TST), as well as to monitor bed times and wake times throughout treatment. The Dysfunctional Beliefs and Attitudes about Sleep (DBAS [41]) measures changes in sleep-disruptive cognitions and beliefs across treatment. The Pittsburgh Sleep Quality Index (PSQI [42]) is a measure of general sleep quality and the Epworth Sleepiness Scale (ESS [43]) measures daytime sleepiness.

Additional secondary outcome measures focus on selfreported psychiatric, psychosocial, and quality of life parameters. Psychiatric symptoms are assessed with the Patient Health Questionnaire 9 (PHQ-9 [44]) for depressive symptoms, the Generalized Anxiety Disorder 7 (GAD7 [45]) for anxiety symptoms, and the PTSD Checklist for DSM-5 (PCL-5 [46]) for symptoms of posttraumatic stress disorder. Fatigue is measured by the Patient-Reported Outcome Measurement Information System (PROMIS) fatigue scale [47]. The PROMIS Global Health Scale [48] measures overall quality of life and the Work and Social Adjustment Scale (WSAS) [49] measures psychosocial functioning. Each of these outcome measures is administered at baseline, post-treatment, 3-month follow-up, and 12month follow-up (see Fig. 1). To the extent possible, the research team attempts to collect outcome measures at the appropriate time points for all participants, even those who drop out of treatment.

\section{Randomization}

Following the diagnostic assessment and confirmation of eligibility, 56 Veterans are randomized in a 1:1 manner to the two treatment groups, BBTI $(N=28)$ or CBTI $(N$ $=28$ ). Randomization is at the patient level, stratified by age (18-64, 65 + years) and if taking a prescription sedative hypnotic medication (yes/no), with a 1:1 allocation using random block sizes of 2,4 , or 6 . Assignments are generated by the study statistician and placed in opaque, sealed envelopes. The study coordinator is responsible for informing the Veterans of their treatment group assignment. The study PI is blinded to participant allocation but may need to be unblinded for clinical reasons (e.g., participant dropout and seeking clinical referral for treatment).

\section{Clinical interventions}

CBTI [50] is a structured treatment that addresses sleeprelated behaviors and cognitions; it is effective in reducing insomnia severity and improving sleep quality, and is the standard non-pharmacological treatment for insomnia in VA. As part of this study, CBTI consists of five in-person sessions (weekly or biweekly) and is delivered by two licensed psychologists, trained in CBTI. The behavioral aspects of the treatment include sleep restriction (to increase the sleep drive and consolidate sleep through decreasing SOL and WASO) and stimulus control (to strengthen the bed as a cue for sleep and remove non-sleep stimuli from the bedroom environment) and may also include relaxation exercises (to reduce physiological arousal). The cognitive component focuses on restructuring or changing maladaptive sleep-related cognitions, such as thoughts that increase cognitive arousal and beliefs that interfere with adherence to the behavioral aspects of insomnia treatment.

BBTI [51] is a structured treatment that focuses on sleep-related behaviors and is effective for the treatment of insomnia. The behavioral aspects of the treatment, similar to those in CBTI, have been shown empirically to improve sleep quality through sleep restriction and stimulus control. Unlike CBTI, there are no cognitive components to treatment. BBTI is delivered over four consecutive weeks, consisting of two in-person sessions (weeks 1 and 3) and two phone sessions (weeks 2 and 4). The therapist manual and participant workbook were adapted for Veterans from the initial treatment protocols [51]. Treatment is delivered by two licensed psychologists, trained in BBTI.

Study clinicians are rated for treatment fidelity and competency using the CBTI rating scale from the VA CBTI 
Therapist Manual. BBTI clinicians are rated on an adapted rating scale. Treatment sessions are rated based on audiorecordings on a monthly basis with a random sample of each clinician rated. If study clinician's ratings are below the cut-off $(<28$, CBTI; $<22$, BBTI $)$ the study PI provides additional training to the clinician. Discontinuation from treatment is voluntary per the participant or if deemed clinically indicated by the study clinicians.

\section{Follow-up assessments}

The self-report measures assessed at baseline are repeated following the end of their intervention (post treatment), at 3 -months post-treatment, and again at 12-months posttreatment.

\section{Hypothesis}

We hypothesize that both BBTI and CBTI significantly reduce insomnia symptoms, per the ISI, from pre- to post-treatment. We also hypothesize that BBTI is a noninferior (i.e., similar) treatment to CBTI based on ISI change scores from pre- to post-treatment.

\section{Data analysis}

To test the effectiveness of BBTI and CBTI, using an "intent-to-treat" approach, we will fit a linear mixed model that tests for the main effects of treatment group (BBTI versus CBTI) and time (baseline, post-treatment, 3-month, and 12-month follow-ups) as well as the interaction of treatment and time. A secondary analysis of treatment effectiveness includes calculating the rates of treatment response and remission, and calculating the number needed to treat (NNT) for BBTI and CBTI [30]. To determine treatment non-inferiority, the $95 \%$ confidence interval (CI) of the mean $\triangle \mathrm{ISI}$ for the BBTI group is compared to a non-inferiority margin (NIM) $[52,53]$. If the $95 \%$ CI is entirely to the right of the NIM, we will have shown noninferiority [54]. The non-inferiority margin (NIM) represents the maximum acceptable difference between $\Delta \mathrm{ISI}_{\mathrm{BBTI}}$ and $\triangle \mathrm{ISI}_{\mathrm{CBTI}}$.

\section{Power analysis}

To achieve adequate power (power $=0.80, \alpha=0.05$ ), a sample size of $n=42$ ( $n=21$ per group) at post-treatment was determined using methods appropriate for non-inferiority trials [55]. Estimation of sample size was informed by the Reliable Change Index (RCI [56]), which was calculated using data from the VA CBTI rollout, a nationwide effort to train providers in the VA to deliver CBTI [29]. The RCI is a metric to help determine if the magnitude of change (e.g., pre- to post-treatment) is statistically reliable. For this comparative effectiveness trial, RCI represents the change on the ISI from pre- to post-treatment (CBTI) that would be expected by chance. An RCI $>1.96$ indicates that the post-test score likely reflects real change (versus the pre-test score) and the change is not due to chance [56]. The RCI used for power analysis, based on the pre- to post-CBTI $\triangle \mathrm{ISI}$, was $\mathrm{RCI}=3.43$. In order to reach $n=42$, our goal is to randomize 56 Veterans ( $n=28$ /group) given that we are estimating $25 \%$ withdrawal/dropout after randomization (based on the VA CBTI rollout $[28,29]$ and BBTI clinical trials [30, 32]).

\section{Aim 2: qualitative needs assessment to identify perceived barriers to, and facilitators of, implementation \\ Data source}

Primary care providers, primary care nurses, and Veterans (from aim 1).

\section{Recruitment and participants}

The target sample is $8-12$ primary care providers (physicians, physician assistants, and nurse practitioners) and 812 primary care nurses (registered nurse care managers and licensed practical nurses) from the study VAMC who treat patients with chronic insomnia. We use purposive sampling so that we can gather a range of perspectives from healthcare providers involved in the care process and Veterans involved in the clinical trial. To the best of our ability, we use the typical case strategy in order to highlight the general experience of primary care providers and nurses who treat patients with insomnia [57]. We also plan to use the snowball strategy so that those who we recruit and interview can help us identify additional similar providers and nurses in primary care who would also be informative [57]. These types of providers are targeted as they represent the front-line clinicians that Veterans typically engage with and report various health problems and symptoms to, including difficulty sleeping. It is valuable to know these providers' thoughts, opinions, and perspectives in order to identify and better understand perceived barriers to, and facilitators of, implementation and to help improve access to care. Recruitment methods of primary care providers and nurses include invitation to participate by the PI based on established relationships through his work as a primary care-mental health integration psychologist. Also, primary care leadership is assisting with recruitment through the broad invitation to providers and nurses to participate in the research study by email. Also targeted for recruitment are 8-12 Veterans from each treatment group in aim 1 who completed at least one treatment session. Similar to the providers, the feedback from Veterans engaged in treatment will provide useful information to help improve access to care. However, the Veteran interview will differ from the provider interview with a focus on their experiences in treatment, opinions and perspectives on the treatment not received, and preferences for care. The goal of recruiting 8-12 per group is to achieve thematic saturation, when data collection and analysis reveals no new themes [58]. If thematic saturation is not achieved with 8 
12 participants in each group, then we will continue with interviews until saturation is reached [58]. The study coordinator or other research staff administers written informed consent; the study coordinator, research staff, or PI conducts the qualitative interviews.

\section{Measures and data collection}

As mentioned above, the goal of this qualitative needs assessment is to identify perceived barriers to, and facilitators of, implementing behavioral treatment of insomnia into the primary care setting. Guiding the discovery and evaluation of implementation barriers is the CFIR [33]. The CFIR was developed with the specific goal of improving Veteran healthcare by implementing research findings into routine clinical practice. It is advantageous for initial implementation efforts as it combines numerous implementation theories and can be applied across a broad range of domains. CFIR is organized into five domains, each with numerous factors that may influence successful implementation; the five domains are: (1) intervention characteristics, including the strength and quality of treatments; (2) outer settings such as external policies and incentives to integrate treatment; (3) inner settings such as the culture of a clinic; (4) individual characteristics such as self-efficacy to engage in, and benefit from, treatment; and (5) the implementation process such as engagement of clinic providers in using a treatment. The CFIR domains and factors act as a guiding framework for implementation research.

The basic structure of the interview guide for the primary care providers and nurses is to introduce them to the basics of BBTI and CBTI and then ask a series of openended questions and discussion items related to CFIR factors, assessing any perceived barriers to, or facilitators of, implementing brief treatments for insomnia in the primary care setting. Veteran interviews focus on their experience in treatment, how it can further be improved, as well as a discussion about the treatment they received versus the treatment they did not.

\section{Hypothesis}

There are no specific a priori hypotheses. However, the goal of this qualitative aim is to identify potential providerand Veteran-level factors, using a CFIR-guided qualitative needs assessment, which may impact successful implementation and integration of behavioral and cognitive behavioral treatments for insomnia in the primary care setting and help to improve access to care.

\section{Data analysis}

Using a constant comparative approach, data collection and analysis will be concurrent. Coding will use the Editing Style [59], which involves an open iterative coding approach that will allow for identification of basic concepts related to the CFIR factors as well as any additional factors that arise.
As each interview is conducted and transcribed, transcripts are reviewed by two coders to identify emergent themes and develop preliminary codebooks based on CFIR coding methods; a codebook will be developed for the providers and nurses and a separate codebook for Veterans [60]. The process involves two coders reading each transcript a number of times to familiarize themselves with the content and categorize the data. The coders will compare newly gathered and previously collected data to identify emergent concepts, categories, themes and relationships in the data and develop an initial set of codes to be applied to subsequent transcripts. As additional transcripts are examined, codes are operationally defined, refined, and agreed upon by the coders. Similar or related codes are collapsed and large codes will, when needed, be separated into more refined and conceptually precise codes. The focus is on identifying specific perceived barriers to, and facilitators of, successful implementation using the CFIR domains and factors. The provider and nurse codebook, and the Veteran codebook are finalized after review of the first four to six transcripts per cohort (providers, nurses, and Veterans); however, we will remain receptive to potential new codes emergent in remaining transcripts [61]. Once the codebooks are compiled and consensus is reached for each cohort, the finalized codebooks are independently applied to all transcripts by the two coders. Throughout codebook development and the coding process, the two coders meet to compare coding and resolve any discrepancies through negotiated consensus [62]. A study team member and expert qualitative methodologist serves as an adjudicator to ensure codebook development consistency and help resolve coding conflicts. Final analysis will allow us to identify common themes within the groups (i.e., provider/nurse and Veteran).

\section{Discussion}

As CBTI continues to be disseminated to providers across the VA, it is increasingly important to better understand barriers to, and facilitators of, successful implementation of insomnia treatment in order to ensure that Veterans have the best access to care. Furthermore, it is important to evaluate the potential for new avenues of treatment delivery, like BBTI, that can benefit Veterans who may not have adequate access to specialty mental health providers trained in CBTI. As described above, this hybrid type I project preliminarily tests the clinical non-inferiority of a briefer, primary care-friendlier treatment, BBTI, versus the current "gold standard" treatment for insomnia, CBTI. Furthermore, utilizing CFIR-guided qualitative interviews with primary care providers and nurses, this project also seeks to identify key perceived barriers to, and facilitators of, implementing BBTI, or similar treatments, into the primary care setting in order to improve access to care. For Veterans, the interviews will help to understand their 
experiences in care and their care preferences. These are two of the strengths of this hybrid type I project.

Additionally, if the clinical trial is successful, and BBTI is shown to be non-inferior to CBTI, there may be a pathway toward broader training of BBTI as a complementary treatment to CBTI, with training focused on primary care staff like nurses and social workers, especially in settings where psychologists are not easily accessible or available (e.g., community-based outpatient clinics). Alternatively, even if BBTI is not found to be non-inferior to CBTI, there are still several directions to pursue. For example, if BBTI is not non-inferior to CBTI but still results in a significant treatment response for some Veterans, BBTI may still be appropriate for widespread dissemination and play an important role in stepped-care treatment for insomnia throughout VA. Also, by conducting a hybrid trial, the process of implementing high-quality, evidence-based practices, like BBTI, may be accelerated by the valuable input gained through qualitative interviews with both providers and Veterans. The qualitative interviews afford the participants the opportunity to provide in-depth information about their perspectives and insights about improving access to care, including advantages, disadvantages, and ways to succeed and the potential pitfalls to avoid from the frontline clinicians, and the open-ended interview structure allows for participant-directed responses. The perspectives of providers and Veterans on how to successfully implement BBTI into primary care settings can help guide the development of methods around identified barriers. Lastly, our multidisciplinary team has the broad spectrum of health services expertise needed to conduct this research, including health psychology, behavioral sleep medicine, implementation science, and qualitative and quantitative methods. The team members can make a substantive contribution to what is known about the implementation of behavioral treatments for insomnia in the primary care setting and improving access to care for Veterans with chronic insomnia.

While this study has potential to improve access to care for Veterans with chronic insomnia, it is not without limitations. The study site is a single, urban VAMC that is currently adequately staffed to manage its Veterans with insomnia. This may limit the generalizability of results for both study aims, not only among other VAMCs but also for the general community. Conducting a multisite hybrid trial may have helped to solve these limitations and may be an appropriate design for future studies to confirm, expand, or explore alternatives based on the current study's findings. In regards to limitations of qualitative research, the goal is not generalizability in a statistical sense, but it is important to assess the ability of the proposed research to generate findings with utility beyond merely describing the specific study settings and sample. Qualitative research has been shown to produce fine-grained and rich descriptive analysis not achievable with purely quantitative approaches, and generate hypotheses and theoretical insights that can be usefully extrapolated, tested, and implemented beyond the specific study settings and sample. Given the current lack of empirical data on BBTI versus CBTI as well as implementing brief behavioral treatments into the primary care setting, we argue that a qualitative approach to identifying providers' perceived barriers to, and facilitators and Veterans' experiences of, treatment and preferences for care, is well justified and will make a significant contribution to the existing knowledge base. We have chosen to include a primary care providers (i.e., physicians, physician assistants, and nurse practitioners), nurses (registered nurse care managers and licensed practical nurses), and Veterans in hopes of increasing variation in the perspectives of valuable stakeholders and maximizing our ability to identify a more exhaustive list of barriers to, and facilitators of, implementation.

CBTI currently has significant support as the recommended first-line treatment for chronic insomnia for adults [34]. Still, there is much work to be done to provide effective and accessible insomnia care to Veterans, as well as the general community, and the results of this hybrid study may help reach those goals.

This project is registered with ClinicalTrials.gov, ID: NCT02724800 (URL: https://clinicaltrials.gov/ct2/show/ NCT02724800).

\section{Trial status}

VAPHS Institutional Review Board (IRB) approval for this study was granted in December 2015. Funding from VA HSR\&D began in April 2016. Recruitment was initiated in May 2016 with the first participant randomized in June 2016. The final participant randomization is expected in January 2019.

\section{Additional file}

Additional file 1: SPIRIT guidelines Checklist. (DOC $120 \mathrm{~kb}$ )

\section{Abbreviations}

BBTI: Brief Behavioral Treatment for Insomnia; CBTI: Cognitive Behavioral Therapy for Insomnia; CFIR: Consolidated Framework for Implementation Research; Cl: Confidence interval; DBAS: Dysfunctional Beliefs and Attitudes about Sleep; DSM-5: Diagnostic and Statistical Manual of Mental Disorders, 5th Edition; DSM-5-SW: STRONG STAR Clinical Interview for DSM-5 Sleep-Wake Disorders; ESS: Epworth Sleepiness Scale; GAD-7: Generalized Anxiety Disorder Scale; IRB: Institutional Review Board; ISI: Insomnia Severity Index; NIM: Non-inferiority margin; NNT: Number needed to treat; PCL-5: PTSD Check List for DSM-5; PI: Principal investigator; PROMIS: Patient-Reported Outcomes Measurement Information System; PSQI: Pittsburgh Sleep Quality Index; PTSD: Posttraumatic Stress Disorder; RCI: Reliable Change Index; SCID5-RV: Structured Clinical Interview for DSM-5, Research Version; SE: Sleep efficiency; SLUMS: St. Louis University Mental Status Examination; SOL: Sleep onset latency; SPIRIT: Standard Protocol Items: Recommendations for Interventional Trials; TIB: Time in bed; TST: Total sleep time; VA: Veterans Affairs; WASO: Wake after sleep onset; WSAS: Work and Social Adjustment Scale 


\section{Acknowledgements}

We would like to acknowledge and thank study consultants (Charles Atwood, Kelly Burkitt, Laura Damschroder, Gretchen Haas, Rachel Manber, Andrew Pomerantz, and Susan Zickmund), clinicians (Alyssa Ford, Jebediah Northern, Danielle Novick, Caitlan Tighe, and Jody Tomko), and research staff (Casey Carl, Molly Daley, and Lisa Lederer) for their contributions and assistance on this project.

\section{Funding}

The study PI and lead author, ADB, and this project, is supported by Career Development Award 13-260 from the US Department of Veterans Affairs, Health Services Research and Development Service. The views expressed are those of the authors and do not necessarily represent the views of the US Department of Veterans Affairs or the United States Government. The study sponsor and funding agency had no role in the design, collection, management, analysis, interpretation, or writing of the report.

\section{Availability of data and materials}

Not applicable

\section{Authors' contributions}

ADB, the PI and lead author, conceptualized the study, and led the writing of the grant application with contributions from his mentorship team: AG, AOY, KLR, and MJC. All authors read and approved the final manuscript.

\section{Ethics approval and consent to participate}

The VA Pittsburgh Healthcare System's Institutional Review Board approval for this study was granted in December 2015. Written informed consent will be obtained from all participants prior to their participation.

\section{Consent for publication}

Not applicable

\section{Competing interests}

The authors declare that they have no competing interests.

\section{Publisher's note}

Springer Nature remains neutral with regard to jurisdictional claims in published maps and institutional affiliations.

\section{Author details}

${ }^{1}$ Center for Health Equity Research and Promotion, VA Pittsburgh Healthcare System, Research Office Building (151R-U), University Drive C, Pittsburgh, PA 15240, USA. ${ }^{2}$ Mental Illness Research, Education and Clinical Center, VA Pittsburgh Healthcare System, Research Office Building (151R-U), University Drive C, Pittsburgh, PA 15240, USA. ${ }^{3}$ Department of Psychiatry, University of Pittsburgh, 3811 O'Hara Street, Pittsburgh, PA 15213, USA. ${ }^{4}$ Department of Biostatistics, Graduate School of Public Health, University of Pittsburgh, Pittsburgh, PA, USA. ${ }^{5}$ Division of General Internal Medicine, Department of Medicine, University of Pittsburgh, Pittsburgh, PA, USA. 'RAND Corporation, 4570 Fifth Avenue, Suite 600, Pittsburgh 15213, PA, USA.

\section{Received: 9 November 2017 Accepted: 29 December 2017}

Published online: 26 January 2018

\section{References}

1. Hoge CW, McGurk D, Thomas JL, Cox AL, Engel CC, Castro CA. Mild traumatic brain injury in U.S. Soldiers returning from Iraq. N Engl J Med. 2008;358:453-63. https://doi.org/10.1056/NEJMoa072972.

2. Lewis V, Creamer M, Failla S. Is poor sleep in veterans a function of posttraumatic stress disorder? Mil Med. 2009;174:948-51.

3. Maher MJ, Rego SA, Asnis GM. Sleep disturbances in patients with posttraumatic stress disorder: epidemiology, impact and approaches to management. CNS Drugs. 2006;20:567-90.

4. Mustafa M, Erokwu N, Ebose I, Strohl K. Sleep problems and the risk for sleep disorders in an outpatient veteran population. Sleep Breath. 2005;9: 57-63. https://doi.org/10.1007/s11325-005-0016-z.

5. Polley M, Frank D, Smith M. National Veteran Sleep Survey: results and findings. Falls Church, VA: VetAdvisor, LLC. 2013;1-6. http://myvetadvisor. com/wp-content/uploads/2013/07/Netadvisor_sleepreport-1.pdf.
6. Troxel WM, Shih RA, Pedersen E, Geyer L, Fisher MP, Griffin BA, et al. Sleep in the Military: Promoting healthy sleep among U.S. servicemembers. 2015; RR-739-OSD:1-282

7. Cozza S, Benedek D, Bradley J, Grieger T, Nam T, Waldrep D. Topics specific to the psychiatric treatment of military personnel, The Iraq War Clinician Guide. Washington, DC: National Center for PTSD, Department of Veterans Affairs; 2004. p. 4-20

8. McCall W. A psychiatric perspective on insomnia. J Clin Psychiatry. 2001;62 Suppl 10:27-32.

9. Taylor DJ, Lichstein KL, Durrence HH, Reidel BW, Bush AJ. Epidemiology of insomnia, depression, and anxiety. Sleep. 2005;28:1457-64.

10. Taylor DJ, Mallory $\amalg$, Lichstein KL, Durrence HH, Riedel BW, Bush AJ. Comorbidity of chronic insomnia with medical problems. Sleep. 2007;30:213-8

11. Bramoweth AD, Germain A. Deployment-related insomnia in military personnel and veterans. Curr Psychiatry Rep. 2013:15:401

12. Bramoweth $A D$, Gregory MP, Walker JD. Clinical characterization of insomnia in the VA: diagnosis and medication use. Sleep. 2013;36:A200.

13. Bramoweth AD, Renqvist JG, Hanusa BH, Walker JD, Germain A, Atwood $C W$, Jr. Identifying the demographic and mental health factors that influence insomnia treatment recommendations within a veteran population. Behav Sleep Med. 2017;1-12. https://doi.org/10.1080/15402002. 2017.1318752

14. Baglioni C, Battagliese G, Feige B, Spiegelhalder K, Nissen C, Voderholzer U, et al. Insomnia as a predictor of depression: a meta-analytic evaluation of longitudinal epidemiological studies. J Affect Disord. 2011;135:10-9. https:// doi.org/10.1016/j.jad.2011.01.011

15. Troxel WM, Buysse DJ, Matthews KA, Kip KE, Strollo PJ, Hall M, et al. Sleep symptoms predict the development of the metabolic syndrome. Sleep. 2010;33:1633-40.

16. Daley M, Morin CM, LeBlanc M, Gregoire J, Savard J. The economic burden of insomnia: direct and indirect costs for individuals with insomnia syndrome, insomnia symptoms, and good sleepers. Sleep. 2009;32:55-64.

17. Martin S. Toward cost-effectiveness analysis in the diagnosis and treatment of insomnia. Sleep Med Rev. 2004;8:63-72. https://doi.org/10.1016/j.smrv. 2003.08.001

18. Stoller M. Economic effects of insomnia. Clin Ther. 1994;16:873-97.

19. Bramoweth AD, Taylor DJ. Chronic insomnia and health care utilization in young adults. Behav Sleep Med. 2012;10:106-21.

20. Leger D, Poursain B. An international survey of insomnia: under-recognition and under-treatment of a polysymptomatic condition. Curr Med Res Opin. 2005;21:1785-92.

21. Wilson SJ, Nutt DJ, Alford C, Argyropoulos SV, Baldwin DS, Bateson AN, et al British Association for Psychopharmacology consensus statement on evidence-based treatment of insomnia, parasomnias and circadian rhythm disorders. J Psychopharmacol. 2010;24:1577-601. https://doi.org/10.1177/ 0269881110379307

22. National Institutes of Health. NIH State-of-the-Science Conference on Manifestations and Management of Chronic Insomnia. 2005. p. 1-105.

23. Kales JD, Kales A, Bixler EO, Soldatos CR, Cadieux RJ, Kashurba GJ, et al. Biopsychobehavioral correlates of insomnia, $\mathrm{V}$ : clinical characteristics and behavioral correlates. Am J Psychiatry. 1984;141:1371-6.

24. Carson S, McDonagh M, Thakurta S, Yen P. Drug class review: newer drugs for insomnia. 2008. p. 1-395

25. Riemann D, Perlis ML. The treatments of chronic insomnia: a review of benzodiazepine receptor agonists and psychological and behaviora therapies. Sleep Med Rev. 2009;13:205-14. https://doi.org/10.1016/j.smrv. 2008.06.001

26. Smith MT, Perlis ML, Park A, Smith MS, Pennington J, Giles DE, et al. Comparative meta-analysis of pharmacotherapy and behavior therapy for persistent insomnia. Am J Psychiatry. 2002;159:5-11.

27. McCrae CS, Bramoweth AD, Williams J, Roth A, Mosti C. Impact of cognitive behavioral treatment for insomnia on health care utilization and costs. J Clin Sleep Med. 2014:10:127-35.

28. Karlin BE, Trockel M, Taylor C, Gimeno J, Manber R. National dissemination of cognitive behavioral therapy for insomnia in veterans: therapist- and patient-level outcomes. J Consult Clin Psychol. 2013;81:912-7.

29. Trockel M, Karlin BE, Taylor CB, Manber R. Cognitive behavioral therapy for insomnia with veterans: evaluation of effectiveness and correlates of treatment outcomes. Behav Res Ther. 2014;53:41-6. https:/doi.org/10.1016/j.brat.2013.11.006.

30. Buysse DJ, Germain A, Moul DE, Franzen PL, Brar LK, Fletcher ME, et al. Efficacy of brief behavioral treatment for chronic insomnia in older adults. Arch Intern Med. 2011;171:887-95. 
31. Germain A, Shear MK, Hall M, Buysse DJ. Effects of a brief behavioral treatment for PTSD-related sleep disturbances: a pilot study. Behav Res Ther. 2007;45:627-32. https://doi.org/10.1016/j.brat.2006.04.009.

32. Germain A, Richardson R, Stocker R, Mammen O, Hall M, Bramoweth AD, et al. Treatment for insomnia in combat-exposed OEF/OIF/OND military veterans: preliminary randomized controlled trial. Behav Res Ther. 2014;61:78-88.

33. Damschroder $L$, Aron DC, Keith RE, Kirsh SR, Alexander JA, Lowery JC. Fostering implementation of health services research findings into practice: a consolidated framework for advancing implementation science. Implement Sci. 2009;4:50. https://doi.org/10.1186/1748-5908-4-50.

34. Qaseem A, Kansagara D, Forciea MA, Cooke M, Denberg TD. Clinical Guidelines Committee of the American College of Physicians. Management of chronic insomnia disorder in adults: a clinical practice guideline from the American College of Physicians. Ann Intern Med. 2016;165:125-33. https:// doi.org/10.7326/M15-2175.

35. STRONG STAR. Clinical Interview for DSM-5 Sleep-Wake Disorders. 2012.

36. Chung F, Subramanyam R, Liao P, Sasaki E, Shapiro C, Sun Y. High STOPBang score indicates a high probability of obstructive sleep apnoea. $\mathrm{Br} J$ Anaesth. 2012;108:768-75.

37. Tariq SH, Tumosa N, Chibnall JT, Perry III MH, Morley JE. Comparison of the Saint Louis University Mental Status Examination and the Mini-mental State Examination for detecting dementia and mild neurocognitive disorder-a pilot study. Am J Geriatr Psychiatry. 2006;14:900-10.

38. First MB, Williams JBW, Karg RS, Spitzer RL. Structured Clinical Interview for the DSM-5, Research Version. Arlington, VA: American Psychiatric Association; 2015.

39. Bastien $\mathrm{CH}$, Vallieres A, Morin CM. Validation of the Insomnia Severity Index as an outcome measure for insomnia research. Sleep Med. 2001;2:297-307.

40. Carney CE, Buysse DJ, Ancoli-Israel S, Edinger JD, Krystal AD, Lichstein KL, et al. The consensus sleep diary: standardizing prospective sleep selfmonitoring. Sleep. 2012;35:287-302. https://doi.org/10.5665/sleep.1642

41. Morin CM, Vallieres A, Ivers H. Dysfunctional Beliefs and Attitudes about Sleep (DBAS): validation of a brief version (DBAS-16). Sleep. 2007;30:1547-54

42. Buysse DJ, Reynolds III CF, Monk TH, Berman SR, Kupfer DJ. The Pittsburgh Sleep Quality Index: a new instrument for psychiatric practice and research. Psychiatry Res. 1989;28:193-213.

43. Johns M. A new method for measuring daytime sleepiness: the Epworth Sleepiness Scale. Sleep. 1991;14:540-5.

44. Kroenke K, Spitzer RL, Williams JB. The PHQ-9: validity of a brief depression severity measure. J Gen Intern Med. 2001;16:606-13. https://doi.org/jgi01114.

45. Spitzer RL, Kroenke K, Williams JB, Lowe B. A brief measure for assessing generalized anxiety disorder: the GAD-7. Arch Intern Med. 2006;166:1092-7. https://doi.org/166/10/1092.

46. Weathers FW, Litz BT, Keane TM, Palmieri PA, Marx BP, Schnurr PP. The PTSD Checklist for DSM-5 (PCL-5). National Center for PTSD. 2013.

47. Reeve BB, Hays RD, Bjorner JB, Cook KF, Crane PK, Teresi JA, et al. Psychometric evaluation and calibration of health-related quality of life item banks: plans for the Patient-Reported Outcomes Measurement Information System (PROMIS). Med Care. 2007;45:S22-31. https://doi.org/10.1097/01.mlr. 0000250483.85507.04.

48. Hays RD, Bjorner JB, Revicki DA, Spritzer KL, Cella D. Development of physical and mental health summary scores from the Patient-reported Outcomes Measurement Information System (PROMIS) global items. Qual Life Res. 2009;18:873-80. https://doi.org/10.1007/s11136-009-9496-9.

49. Mundt JC, Marks IM, Shear MK, Greist JH. The Work and Social Adjustment Scale: a simple measure of impairment in functioning. Br J Psychiatry. 2002; 180:461-4.

50. Manber R, Friedman L, Siebern AT, Carney C, Edinger J, Epstein D, et al. Cognitive behavioral therapy for insomnia in Veterans: therapist manual. Washington, DC: U.S. Department of Veterans Affairs; 2014.

51. Troxel WM, Germain A, Buysse DJ. Clinical management of insomnia with brief behavioral treatment (BBTI). Behav Sleep Med. 2012;10:266-79. https:// doi.org/10.1080/15402002.2011.607200.

52. Christensen E. Methodology of superiority vs. equivalence trials and noninferiority trials. J Hepatol. 2007:46:947-54. https://doi.org/10.1016/j.jhep. 2007.02.015.

53. Jones $B$, Jarvis $P$, Lewis JA, Ebbutt AF. Trials to assess equivalence: the importance of rigorous methods. BMJ. 1996;313:36-9.

54. Hermens ML, van Hout HP, Terluin B, Adèr HJ, Penninx BW, van Marwijk $H W$, et al. Clinical effectiveness of usual care with or without antidepressant medication for primary care patients with minor or mild-major depression: a randomized equivalence trial. BMC Med. 2007:5:36.

55. Julious SA. Tutorial in biostatistics: sample sizes for clinical trials with normal data. Statist Med. 2004:23:1921-86. https://doi.org/10.1002/sim.1783.

56. Jacobson NS, Truax P. Clinical significance: a statistical approach to defining meaningful change in psychotherapy research. J Consult Clin Psychol. 1991;59:12-9.

57. Palinkas LA, Horwitz SM, Green CA, Wisdom JP, Duan N, Hoagwood K. Purposeful sampling for qualitative data collection and analysis in mixed method implementation research. Adm Policy Ment Health. 2015;42:533-44. https://doi.org/10.1007/s10488-013-0528-y.

58. Guest $\mathrm{G}$, Bunce A, Johnson L. How many interviews are enough? An experiment with data saturation and variability. Field Methods. 2006;18:59-82.

59. Crabtree BF, Miller WL. Doing qualitative research. 2nd ed. Thousand Oaks: SAGE; 1999.

60. CFIR Research Team. Qualitative data. In: Consolidated framework for implementation research. 2014. http://www.cfirguide.org/qual.html. Accessed 30 March 2017.

61. Strauss A, Corbin JM. Basics of qualitative research. 2nd ed. Thousand Oaks: SAGE; 1998.

62. Bradley EH, Curry LA, Devers KJ. Qualitative data analysis for health services research: developing taxonomy, themes, and theory. Health Serv Res. 2007; 42:1758-72; https://doi.org/HESR684.

\section{Submit your next manuscript to BioMed Central and we will help you at every step:}

- We accept pre-submission inquiries

- Our selector tool helps you to find the most relevant journal

- We provide round the clock customer support

- Convenient online submission

- Thorough peer review

- Inclusion in PubMed and all major indexing services

- Maximum visibility for your research

Submit your manuscript at www.biomedcentral.com/submit
) Biomed Central 